Hellany Karolliny Pinho Ribeiro ${ }^{a}$ (iD) https://orcid.org/0000-0002-3157-7924

José Diego Marques Santos ${ }^{\mathrm{a}}$ (iD) https://orcid.org/0000-0001-7973-7678

Monaliza de Goes e Silva ${ }^{a}$

(iD) https://orcid.org/0000-0001-5830-3897

Flávia Daniele de Alencar Medeiro ${ }^{\mathrm{a}}$ (iD) https://orcid.org/0000-0002-2980-629X

Márcia Astrês Fernandes ${ }^{a}$

(iD) https://orcid.org/0000-0001-9781-0752

aniversidade Federal do Piauí (UFPI). Departamento de Enfermagem. Teresina, PI, Brasil.

Contato:

Hellany Karolliny Pinho Ribeiro

E-mail:

hellanyribeiro@hotmail.com

Os autores declaram que o estudo não foi subvencionado e que não há conflitos de interesses.

Os autores informam que o trabalho não é baseado em tese ou dissertação e não foi apresentado em eventos científicos.

\section{Transtornos de ansiedade como causa de afastamentos laborais}

\author{
Anxiety disorders as a cause of work absenteeism
}

\section{Resumo}

Introdução: transtornos mentais e comportamentais (TMC) estão entre as principais causas de perda de dias de trabalho. Os transtornos de ansiedade são a segunda causa dos afastamentos laborais. Objetivo: identificar e analisar as evidências disponíveis na literatura sobre afastamentos do trabalho por transtornos de ansiedade. Métodos: revisão integrativa da literatura, sem limite de ano de publicação, com descritores controlados, realizada nas bases de dados Literatura Latino-Americana e do Caribe em Ciências da Saúde (Lilacs), Medical Literature Analysis and Retrieval System Online (Medline) e Base de Dados em Enfermagem (BDENF). Resultados: a seleção final consistiu em 13 artigos, sendo o mais antigo publicado em 2006 e o mais recente em 2016. Os afastamentos por transtornos de ansiedade tiveram como principais fatores de risco as condições e ambiente de trabalho, com impacto negativo, tanto para a saúde do trabalhador como para o empregador. Discussão: as evidências apontam para uma alta prevalência dos transtornos ansiosos como causa de afastamentos do trabalho e da alta demanda de custos elevados com auxíliodoença. Conhecer o perfil de adoecimento do trabalhador contribui para identificar os fatores de risco para TMC e auxilia na proposição de estratégias de intervenção direcionadas a reduzir o afastamento laboral.

Palavras-chave: transtornos de ansiedade; saúde do trabalhador; licença médica.

\begin{abstract}
Introduction: mental and behavioral disorders (MBD) are among the main causes of lost workdays. Anxiety disorders are the second cause of work absenteism. Objective: to identify and analyze evidence available in literature about work absenteism due to anxiety disorders. Methods: integrative literature review, with no publication year limits and with controlled subject headings, using the following databases: Latin American and Caribbean Literature on Health Sciences (Lilacs), Medical Literature Analysis and Retrieval System Online (Medline) and Nursing Database (BDENF). Results: the final selection consisted of 13 articles, the oldest published in 2006 and the most recent in 2016. Work absences due to anxiety disorders had as main risk factors the environment and working conditions, with a negative impact for both, the worker's and the employer's health. Discussion: evidence indicates a high prevalence of anxiety disorders as cause of work absenteeism and of the high demand for increasing social security costs. Knowing the worker's illness profile contributes to identify the MBD risk factors, and helps in proposing intervention strategies aimed at reducing work absenteism.
\end{abstract}

Keywords: anxiety disorders; occupational health; sick leave. 


\section{Introdução}

No início dos anos 2000, a Organização Mundial da Saúde (OMS) e a Organização Internacional do Trabalho (OIT) previram o crescimento da incidência de problemas relacionados à saúde mental e alertaram sobre o impacto desse aumento na população trabalhadora: queda de produtividade, afastamento laboral, redução da capacidade funcional e de trabalho, exclusão social e estigmatização de trabalhadores ${ }^{1}$.

Profundas transformações ocorreram nos ambientes e nas condições de trabalho. Mudanças nas condições de emprego na economia global ao longo de 30 anos levaram ao aumento da insegurança no emprego e outros riscos para a organização do trabalho. Esses riscos podem desempenhar um papel na criação e manutenção de disparidades na saúde dos trabalhadores, a depender de posição socioeconômica, gênero, raça e etnia. Deste modo, por conta dos novos sistemas de produção, vivenciam-se condições de trabalho adversas, como redução da oferta de vagas, terceirização, privatização do setor público e das novas ofertas de empregos oriundos da inovação tecnológica ${ }^{2}$.

Como consequência dessas transformações, modificou-se o perfil de morbidade dos agravos à saúde relacionados ao trabalho, resultando em doenças ocupacionais, com destaque para o adoecimento mental. Uma preocupação crescente para a Saúde do Trabalhador são os transtornos mentais e comportamentais (TMC) decorrentes de situações do processo do trabalho ${ }^{3}$.

Os TMC caracterizam-se por alterações clínicas e comportamentais significativas, funcionamento com prejuízo ou qualquer associação destes. Acarretam sofrimento relevante e danos em várias áreas do funcionamento mental, podendo advir de fatores orgânicos, sociais, genéticos, químicos ou psicológicos ${ }^{4}$. Estima-se que $10 \%$ dos adultos manifestem tais condições e que $25 \%$ da população mundial manifeste pelo menos um transtorno mental ao longo da vida. Atualmente os TMC se encontram dentre as principais causas de perda de dias de trabalho ${ }^{5}$.

Nos últimos anos os TMC se mantiveram como a terceira causa de afastamentos laborais com auxílio-doença no Instituto Nacional do Seguro Social (INSS). Possivelmente isso se deve às condições de exposição crônica a estressores psicossociais desfavoráveis no trabalho, associados a queixas psicossomáticas, sintomas psiquiátricos e mudanças no bem-estar ${ }^{6}$.

Estudos brasileiros apontam que tais aspectos afetam a saúde e a qualidade de vida, já que demandam dos profissionais imediatismos resolutivos e atualizações constantes, afetando o ritmo e a capacidade de assimilação das tarefas. Assim, o controle do trabalhador sobre a atividade exercida é sobrepujado pelas demandas que lhe são impostas e configuram risco adicional na direção do adoecimento físico e psicológico $^{7-9}$.

O afastamento decorrente de doenças é um problema de saúde pública e gera impactos sociais, organizacionais e individuais. Seu monitoramento e a avaliação sistemática podem subsidiar a tomada de decisão gerencial, assim como o aperfeiçoamento de políticas de recursos humanos, programas de prevenção à saúde do trabalhador e a melhoria da qualidade de vida no trabalho ${ }^{10}$.

Dentre os TMC, os transtornos de ansiedade, ou ansiosos, são aqueles em que as características da ansiedade são desproporcionais em relação ao estímulo, interferindo na qualidade de vida. Dentre essas características pode haver um sentimento de medo vago, que se manifesta como tensão decorrente de uma antecipação de perigo ${ }^{11}$.

Os transtornos de ansiedade estão entre as categorias mais prevalentes de doenças mentais. Quando não tratados, os sintomas de ansiedade persistem e estão associados a deficiências significativas no funcionamento, má qualidade de vida e um enorme fardo econômico. São particularmente importantes no contexto de conflitos mundiais recentes e contínuos, pois os fatores ambientais podem ter forte impacto na ansiedade e no desenvolvimento de distúrbio do estresse, particularmente o pós-traumático ${ }^{12}$.

Santana et al. ${ }^{13}$ reiteram os transtornos ansiosos como segunda causa maior de afastamentos laborais dentre os TMC. Também ratificam que a ocorrência desse tipo de transtorno está relacionada aos estressores psicossociais desfavoráveis, que incluem ambientes de trabalho com pouco apoio social, excesso de trabalho, recompensas inadequadas ao esforço do trabalhador, comprometimento individual excessivo e más condições dos ambientes e dos processos de trabalho.

Assim, considerando a relevância do tema na atualidade, por se configurar como um problema de saúde pública, este estudo objetivou identificar e analisar as evidências disponíveis na literatura sobre os afastamentos do trabalho por transtornos de ansiedade.

\section{Métodos}

Revisão integrativa da literatura ${ }^{14}$, com a finalidade de reunir, sintetizar, sistematizar e ordenar 
resultados de pesquisas sobre transtornos de ansiedade como causa de afastamento laboral.

Para operacionalizar a revisão, foram realizadas as seguintes etapas: desenvolvimento da questão norteadora; busca dos estudos primários nas bases de dados; avaliação dos estudos selecionados; extração de dados dos estudos; análise e síntese dos resultados e apresentação da revisão. A questão norteadora foi: quais as evidências disponíveis na literatura científica sobre afastamentos laborais por transtornos de ansiedade?

A coleta de dados foi realizada em julho de 2016. Para a seleção dos artigos foram consultadas as bases de dados Literatura Latino-Americana e do Caribe em Ciências da Saúde (Lilacs), Medical Literature Analysis and Retrieval System Online (Medline) e Base de Dados em Enfermagem (BDENF). Os descritores controlados foram combinados da seguinte forma para Lilacs e BDENF: "transtornos de ansiedade AND saúde do trabalhador OR licença médica”. Na Medline, a busca foi norteada por descritores Medical Subject Headings $(\mathrm{MeSH})$ : "anxiety disorders AND occupational health OR sick leave".

Os critérios de inclusão para a pré-seleção dos estudos foram: textos em inglês, português ou espanhol, publicados em periódicos e que abordassem afastamentos do trabalho por transtornos de ansiedade. Os critérios de exclusão foram: artigos não primários, como os de opinião e revisões, e aqueles que, após leitura na íntegra, não responderam ao objetivo desta revisão. Não foi estabelecido limite quanto ao ano de publicação dos artigos.

A seleção dos estudos foi iniciada com a leitura minuciosa de títulos e resumos, incluindo-se aqueles que atendiam aos critérios. Para a seleção final dos artigos foi realizada a leitura integral, identificando-se aqueles que apresentavam dados relacionados a afastamentos do trabalho por transtornos de ansiedade.

Para a análise dos artigos selecionados, foi utilizado um instrumento de coleta e síntese dos dados, elaborado pelos próprios autores, com o propósito de extrair, organizar e sumarizar as informações e facilitar a formação do banco de dados. Os tópicos de interesse abordados no instrumento foram: autores, título do artigo, ano de publicação, idioma, origem do periódico, local onde foi feita a pesquisa, resultados e conclusões. O processo de identificação, seleção e inclusão dos estudos primários se deu em três etapas.

Na primeira etapa foi realizada a leitura de títulos e a retirada dos artigos duplicados. Assim, do total de 1.350 artigos, foram selecionados 232. Na segunda etapa, procedeu-se à leitura dos títulos e resumos dos 232 artigos, à luz dos critérios de inclusão. Assim, foram selecionados 23 artigos.

Na terceira etapa realizou-se a leitura na íntegra desses 23 artigos, sendo retirados 10 que não atendiam aos critérios de inclusão e não respondiam à questão norteadora desta revisão, de modo que ao final restaram 13 artigos. A Figura 1 ilustra o processo de seleção dos artigos desta revisão integrativa.

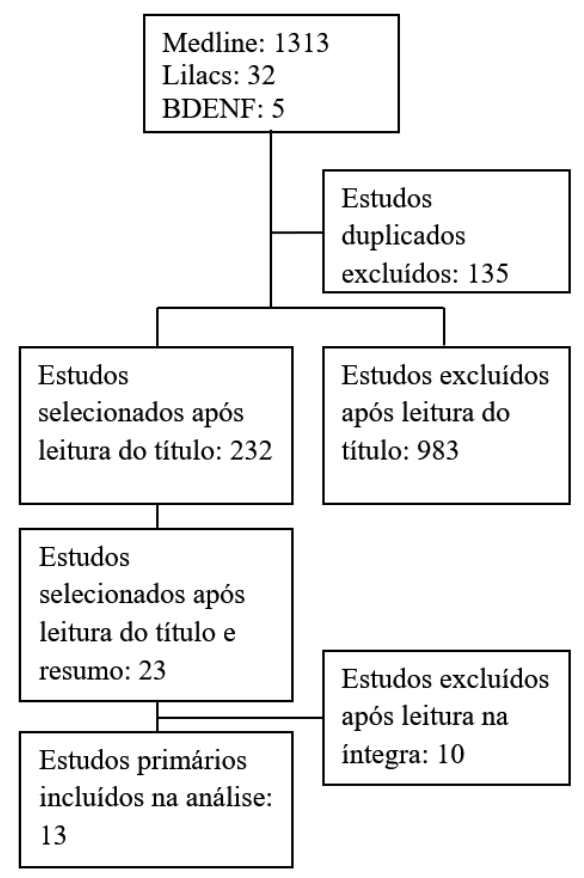

Figura 1 Fluxograma de seleção dos artigos da revisão integrativa

\section{Resultados}

O corpus final consistiu em 13 artigos (Quadro 1), sendo o mais antigo publicado em 2006 e o mais recente em 2016.

Em relação ao idioma, 11 artigos foram publicados em periódicos internacionais, sendo 10 deles em inglês e um em espanhol. Os outros dois foram publicados em português, em periódicos nacionais.

Quanto à localização, os estudos foram realizados no Reino Unido (3), na Holanda (3), Finlândia (1), Cracóvia (1), Alemanha (1), França (1) e Espanha (1). Dois estudos foram realizados no Brasil, sendo um em Minas Gerais e outro na Paraíba.

Os delineamentos mais frequentes foram o estudo de coorte, com 10 artigos, e o estudo transversal, com 3 . 
Quadro 1 Síntese das informações relevantes extraídas dos artigos selecionados, com base no objetivo deste estudo de revisão

\begin{tabular}{|c|c|c|c|}
\hline Autores / Ano & $\begin{array}{c}\text { Idioma/Origem } \\
\text { Local }\end{array}$ & Principais resultados & Conclusões \\
\hline $\begin{array}{l}\text { Nieuwenhuijsen } \\
\text { et al. }(2006)^{15}\end{array}$ & $\begin{array}{l}\text { Inglês/ } \\
\text { Escandinávia } \\
\text { Finlândia }\end{array}$ & $\begin{array}{l}\text { Uma coorte de } 188 \text { funcionários em licença por } \\
\text { transtornos mentais, dentre eles os transtornos } \\
\text { de ansiedade, foi acompanhada por } 1 \text { ano. O } \\
\text { tempo mais longo até o retorno ao trabalho foi } \\
\text { associado a: idade superior a } 50 \text { anos, expectativa } \\
\text { de ausência maior que } 3 \text { meses, menor nível } \\
\text { educacional e diagnóstico de transtorno de } \\
\text { ansiedade. }\end{array}$ & $\begin{array}{l}\text { Uma regra de predição baseada em quatro } \\
\text { variáveis simples - idade, duração do } \\
\text { afastamento, nível educacional e diagnóstico } \\
\text { - pode ser usada por médicos ocupacionais } \\
\text { para identificar casos desfavoráveis e para } \\
\text { prever a duração da ausência no trabalho. }\end{array}$ \\
\hline $\begin{array}{l}\text { Rymaszewska, } \\
\text { Dziełak e Kiejna } \\
(2007)^{16}\end{array}$ & $\begin{array}{l}\text { Inglês/Polônia } \\
\text { Cracóvia }\end{array}$ & $\begin{array}{l}\text { O número de pessoas que sofrem de transtornos } \\
\text { mentais e recebem pensões por invalidez está } \\
\text { cada vez maior. Os jovens são os mais acometidos. } \\
\text { Pessoas que sofrem de distúrbios afetivos e de } \\
\text { ansiedade são o grupo mais comum que recebe } \\
\text { benefícios. }\end{array}$ & $\begin{array}{l}\text { A atribuição de pensão por invalidez a } \\
\text { pessoas que sofrem de distúrbios ansiosos, } \\
\text { bem como por incapacidade para o trabalho, } \\
\text { demanda custos elevados para o país. }\end{array}$ \\
\hline $\begin{array}{l}\text { Andrea et al. } \\
(2009)^{17}\end{array}$ & $\begin{array}{l}\text { Inglês/Estados } \\
\text { Unidos } \\
\text { Holanda }\end{array}$ & $\begin{array}{l}\text { Ansiedade e depressão são prevalentes entre os } \\
\text { funcionários e estão associadas à funcionalidade. } \\
\text { A incidência cumulativa de } 23 \text { meses para } \\
\text { ansiedade e depressão foi de } 4,6 \text { e } 3,3 \% \\
\text { respectivamente. Altas exigências de trabalho, } \\
\text { baixo apoio social e insegurança no emprego } \\
\text { foram preditivos para os transtornos ansiosos } \\
\text { e para o aumento dos casos de afastamento } \\
\text { decorrente. }\end{array}$ & $\begin{array}{l}\text { As características do trabalho são preditores } \\
\text { significativos para o início da ansiedade } \\
\text { e depressão na população trabalhadora. } \\
\text { Modificações no ambiente de trabalho } \\
\text { reduzem tanto ansiedade quanto sintomas } \\
\text { depressivos entre os funcionários, diminuindo } \\
\text { o número de casos de afastamentos. }\end{array}$ \\
\hline $\begin{array}{l}\text { Siano, Ribeiro e } \\
\text { Ribeiro }(2010)^{18}\end{array}$ & $\begin{array}{l}\text { Português/Rio } \\
\text { de Janeiro } \\
\text { Minas Gerais }\end{array}$ & $\begin{array}{l}\text { Transtornos depressivos leves ou moderados } \\
\text { e transtornos persistentes do humor }(39,6 \%) \\
\text { e os transtornos de ansiedade }(34,5 \%) \text { foram } \\
\text { os diagnósticos mais frequentes nos registros } \\
\text { periciais de segurados do INSS requerentes de } \\
\text { auxílio-doença apresentando transtorno mental. } \\
\end{array}$ & $\begin{array}{l}\text { Transtornos de humor e de ansiedade são } \\
\text { descritos como os transtornos mentais mais } \\
\text { frequentemente encontrados na população e } \\
\text { os mais registrados como motivos de ausência } \\
\text { no trabalho. }\end{array}$ \\
\hline $\begin{array}{l}\text { Muschalla, } \\
\text { Linden e } \\
\text { Olbrich }(2010)^{19}\end{array}$ & $\begin{array}{l}\text { Inglês/Estados } \\
\text { Unidos } \\
\text { Reino Unido }\end{array}$ & $\begin{array}{l}\text { A ansiedade relacionada ao trabalho, em } \\
\text { contraste com a ansiedade geral, é por sua própria } \\
\text { natureza associada a problemas de participação } \\
\text { no trabalho, e foi significativamente relacionada à } \\
\text { duração da licença por doença. }\end{array}$ & $\begin{array}{l}\text { A relação entre trabalho e ansiedade é } \\
\text { importante para entender a licença por } \\
\text { doença e aparece como um fenômeno } \\
\text { multidimensional e clinicamente importante. }\end{array}$ \\
\hline $\begin{array}{l}\text { Henderson et al. } \\
(2011)^{20}\end{array}$ & $\begin{array}{l}\text { Inglês/Reino } \\
\text { Unido } \\
\text { Reino Unido }\end{array}$ & $\begin{array}{l}\text { Os transtornos mentais são os motivo mais } \\
\text { comuns para a ausência no trabalho em longo } \\
\text { prazo. A perda associada de produtividade e } \\
\text { o pagamento de benefícios por incapacidade } \\
\text { representam um fardo substancial sobre as } \\
\text { economias de muitos países desenvolvidos. } \\
\text { Isso se relaciona predominantemente com a } \\
\text { depressão e ansiedade. }\end{array}$ & $\begin{array}{l}\text { O ambiente e as condições de trabalho } \\
\text { são fatores de risco que contribuem para } \\
\text { o surgimento de transtornos mentais e } \\
\text { consequentemente o afastamento do } \\
\text { trabalho. }\end{array}$ \\
\hline $\begin{array}{l}\text { Flach et al. } \\
(2012)^{21}\end{array}$ & $\begin{array}{l}\text { Inglês/Países } \\
\text { Baixos } \\
\text { Holanda }\end{array}$ & $\begin{array}{l}\text { Burnout, depressão e transtorno de ansiedade } \\
\text { estão associados a uma maior duração da licença } \\
\text { por doença. Fatores como sexo feminino, função } \\
\text { exercida no trabalho e risco psicossocial de } \\
\text { moderado a alto também estão associados com a } \\
\text { duração da licença médica. }\end{array}$ & $\begin{array}{l}\text { O diagnóstico médico tem forte relação } \\
\text { com o momento do primeiro retorno ao } \\
\text { trabalho e a duração dos períodos de licença } \\
\text { por transtornos mentais, mas a influência } \\
\text { de fatores demográficos não deve ser } \\
\text { negligenciada. }\end{array}$ \\
\hline $\begin{array}{l}\text { Romero et al. } \\
(2013)^{22}\end{array}$ & $\begin{array}{l}\text { Espanhol/ } \\
\text { Espanha } \\
\text { Madri }\end{array}$ & $\begin{array}{l}\text { A duração média da incapacidade temporal por } \\
\text { contingências comuns (ITCC) nos trastornos } \\
\text { de ansiedade foi de } 83 \text { dias. Os trabalhadores } \\
\text { afastados possuem frequentemente idade } \\
\text { superior a } 35 \text { anos, menor nível de estudos e } \\
\text { apresentam comorbidade. }\end{array}$ & $\begin{array}{l}\text { Os trastornos de ansiedade são associados a } \\
\text { períodos maiores de ITCC em relação a outras } \\
\text { doenças. Variáveis demográficas, laborais } \\
\text { e clínicas presentes na avaliação inicial } \\
\text { identificam grupos com maior risco de que } \\
\text { a ITCC se prolongue, e requerem estratégias } \\
\text { orientadas a facilitar a reincorporação laboral. }\end{array}$ \\
\hline
\end{tabular}

(Continua) 
Quadro 1 Continuação...

\begin{tabular}{|c|c|c|c|}
\hline Autores / Ano & $\begin{array}{c}\text { Idioma/Origem } \\
\text { Local }\end{array}$ & Principais resultados & Conclusões \\
\hline $\begin{array}{l}\text { Wedegaertner et } \\
\text { al. }(2013)^{23}\end{array}$ & $\begin{array}{l}\text { Inglês/Reino } \\
\text { Unido } \\
\text { Alemanha }\end{array}$ & $\begin{array}{l}\text { Ansiedade e depressão são os distúrbios } \\
\text { psiquiátricos mais comuns e causam o aumento } \\
\text { da quantidade de licença por doença na maioria } \\
\text { dos países desenvolvidos. Além disso, foram } \\
\text { associadas ao aumento do risco de aposentadoria } \\
\text { por incapacidade permanente, bem como da } \\
\text { mortalidade em uma coorte de trabalhadores } \\
\text { alemães. }\end{array}$ & $\begin{array}{l}\text { Os diagnósticos de depressão/ansiedade são } \\
\text { os que mais demandam licença médica por } \\
\text { doença e aumentam o risco de aposentadoria } \\
\text { antecipada, sugerindo um grupo-alvo para o } \\
\text { desenvolvimento de intervenções. }\end{array}$ \\
\hline $\begin{array}{l}\text { Combs e } \\
\text { Markman } \\
(2014)^{24}\end{array}$ & $\begin{array}{l}\text { Inglês/Estados } \\
\text { Unidos } \\
\text { Reino Unido }\end{array}$ & $\begin{array}{l}\text { Transtornos de ansiedade são os diagnósticos } \\
\text { psiquiátricos mais comuns nos Estados Unidos. } \\
\text { De } 965 \text { pessoas selecionadas no estudo, 19,5\% } \\
\text { apresentaram pelo menos um transtorno de } \\
\text { ansiedade. Essas condições estão associadas } \\
\text { a elevadas taxas de desemprego, períodos de } \\
\text { afastamento do trabalho, diminuição do bem- } \\
\text { estar e maior dependência da assistência pública. }\end{array}$ & $\begin{array}{l}\text { Pacientes com transtornos de ansiedade } \\
\text { podem apresentar sintomas clinicamente } \\
\text { inexplicáveis, fazendo com que o diagnóstico } \\
\text { correto seja um desafio. Além disso, dados } \\
\text { os custos, as consequências do afastamento } \\
\text { decorrente e a carga financeira associada, } \\
\text { identificar e tratar a ansiedade são } \\
\text { imperativos. }\end{array}$ \\
\hline $\begin{array}{l}\text { Niedhammer, } \\
\text { Malard e } \\
\text { Chastang } \\
(2015)^{25}\end{array}$ & $\begin{array}{l}\text { Inglês/Reino } \\
\text { Unido } \\
\text { França }\end{array}$ & $\begin{array}{l}\text { As demandas psicológicas, a baixa recompensa, } \\
\text { as demandas emocionais e a insegurança } \\
\text { no emprego são preditivas de distúrbios de } \\
\text { ansiedade. Quanto mais frequente a exposição } \\
\text { a esses fatores, maior o risco de distúrbios de } \\
\text { ansiedade e, consequentemente, afastamento do } \\
\text { trabalho. }\end{array}$ & $\begin{array}{l}\text { Fatores de trabalho psicossocial, clássicos e } \\
\text { emergentes, foram preditivos de depressão e } \\
\text { ansiedade com associações dose-resposta em } \\
\text { termos de frequência de exposição e duração } \\
\text { do período de afastamento. }\end{array}$ \\
\hline $\begin{array}{l}\text { Batista et al. } \\
(2016)^{26}\end{array}$ & $\begin{array}{l}\text { Português/Rio } \\
\text { de Janeiro } \\
\text { Paraíba }\end{array}$ & $\begin{array}{l}\text { A maior parte da amostra foi composta por } \\
\text { docentes do sexo feminino, com faixa etária entre } \\
40 \text { e } 49 \text { anos. Os transtornos de ansiedade foram } \\
\text { uma das principais causas de afastamentos dos } \\
\text { profissionais no estudo. }\end{array}$ & $\begin{array}{l}\text { São necessárias novas investigações } \\
\text { que possam analisar a gravidade dessa } \\
\text { problemática. Nesta perspectiva, deve-se } \\
\text { elaborar intervenções que considerem } \\
\text { as condições de trabalho como um risco } \\
\text { para o surgimento de transtornos mentais } \\
\text { e consequentemente para a ausência no } \\
\text { trabalho. }\end{array}$ \\
\hline $\begin{array}{l}\text { Den Boeft et al. } \\
(2016)^{27}\end{array}$ & $\begin{array}{l}\text { Inglês/Reino } \\
\text { Unido } \\
\text { Holanda }\end{array}$ & $\begin{array}{l}\text { Sintomas físicos inexplicados são altamente } \\
\text { prevalentes e podem afetar o funcionamento do } \\
\text { trabalho. Foram associados à incapacidade e ao } \\
\text { afastamento em curto e longo prazo ao longo de } \\
2 \text { anos. Para distúrbios depressivos, transtornos } \\
\text { de ansiedade e características do trabalho, as } \\
\text { associações permaneceram significativas. }\end{array}$ & $\begin{array}{l}\text { Sintomas físicos inexplicados foram } \\
\text { associados com o afastamento laboral } \\
\text { mesmo depois de relacioná-los a distúrbios } \\
\text { depressivos, transtornos de ansiedade e } \\
\text { características do trabalho. Isso sugere que } \\
\text { a identificação precoce desses sintomas e o } \\
\text { gerenciamento adequado são importantes. }\end{array}$ \\
\hline
\end{tabular}

\section{Discussão}

Os resultados desta revisão indicam que as evidências disponíveis na literatura sobre os afastamentos laborais por transtornos de ansiedade são relativamente recentes, visto que o artigo mais antigo selecionado para este estudo foi publicado no ano de 2006. É possível identificar também oscilações no número de artigos sobre o tema publicados na última década. Quanto à origem dos estudos selecionados, a maioria foi produzida internacionalmente, o que indica possível escassez de estudos nacionais sobre a temática em questão.

Os transtornos de ansiedade interferem significativamente na vida do trabalhador e daqueles com quem ele convive, comprometendo suas atividades, seus relacionamentos sociais e outras esferas da vida. Apresentam baixos índices de remissão espontânea e tendem a se cronificar, quando não tra$\operatorname{tados}^{28}$. Além disso, são incapacitantes, tendo sido verificadas maiores taxas de afastamento entre aqueles que padecem de tais morbidades. Se a ocorrência for precoce, menores serão as chances de iniciarem, prosseguirem ou se manterem ativos numa profissão. Ademais, os sintomas de ansiedade em trabalhadores foram preditores de desemprego nos anos seguintes $^{29}$.

Soma-se a isso o fato de a saúde dos trabalhadores ser, por vezes, negligenciada tanto pelos empregadores como pelos próprios profissionais, que 
evitam o afastamento para não resultar uma perda salarial, associada ao sentimento de não poder adoecer. Assim, muitas vezes as sobrecargas de trabalho passam despercebidas ou são ignoradas pelos próprios trabalhadores ${ }^{30}$.

Uma associação longitudinal entre sintomas físicos inexplicados e funcionamento do trabalho, realizada em um estudo com 1.887 participantes na Holanda, mostrou-se prevalente $(52,8 \%)$ e pode afetar o funcionamento do trabalho. Isso foi associado à incapacidade e ao afastamento do trabalhador em curto e longo prazo, ao longo de 2 anos, por distúrbios depressivos e transtornos de ansiedade ${ }^{27}$.

Estudo prospectivo realizado também na Holanda, baseado em 3.707 funcionários cujas atividades desempenhadas foram analisadas, apontou que altas exigências do trabalho, baixo apoio social e insegurança no emprego aumentam significativamente o risco de ansiedade e depressão subsequentes. Ademais, quanto mais frequente a exposição a esses fatores, maiores são as chances de o trabalhador apresentar algum tipo de transtorno de ansiedade e, consequentemente, afastar-se do trabalho ${ }^{17}$.

Outra pesquisa, com 4.717 trabalhadores franceses, avaliou as condições e o ambiente de trabalho que predispunham ao adoecimento mental por ansiedade, apontando baixa recompensa salarial, conflitos interprofissionais, tensões com o público para o qual prestavam atendimento e o desequilíbrio entre trabalho e lazer como fatores que aumentam o risco de ansiedade e depressão ${ }^{25}$.

Duas análises, uma realizada na Polônia ${ }^{16}$ e outra na Alemanha ${ }^{23}$, buscaram dados sobre licenças por doenças em trabalhadores, constatando que ansiedade e depressão são os distúrbios psiquiátricos mais comuns e as principais causas de aumento da quantidade de licença por doença na maioria dos países desenvolvidos. Tais distúrbios também estão implicados numa mortalidade crescente e foram associados ao aumento do risco de aposentadoria por incapacidade permanente.

Dados de uma pesquisa realizada em base de dados holandesa ${ }^{21}$ e outro estudo que objetivou investigar a ansiedade relacionada ao trabalho ${ }^{19}$ evidenciaram que os transtornos de ansiedade estão associados a uma maior duração da licença por doença. Este último ainda acrescentou que a licença por doença aparece como um fenômeno multidimensional e clinicamente importante ${ }^{19}$. Corroborando este achado, um estudo retrospectivo de registros de perícias iniciais realizadas em agências da Previdência Social de Juiz de Fora, Minas Gerais, mostrou que os transtornos de ansiedade foram um dos diagnósticos mais frequentes (34,5\%) nos registros periciais de segurados do INSS requerentes de auxílio-doença apresentando transtorno mental ${ }^{18}$.

Em um estudo de coorte com 188 funcionários, dentre os quais 102 eram professores, em licença por doença com transtornos mentais comuns, seguidos por 1 ano em uma pesquisa realizada na Finlândia, foi observado que o tempo mais longo de afastamento do trabalho era daqueles com idade superior a 50 anos, que possuíam menor nível educacional e diagnóstico de transtorno de ansiedade, com expectativa de ausência no trabalho superior a 3 meses $^{15}$.

A propósito disso, um menor nível de instrução pode estar relacionado à maior probabilidade de ausência do trabalho por doenças ocupacionais devido às atividades exercidas e, algumas vezes, à limitação em compreender orientações e instruções. Além disso, para suprir necessidades financeiras, aqueles com baixa escolaridade possuem geralmente mais de um vínculo empregatício, o que contribui para um maior desgaste físico e psicológico, bem como para reduzir o tempo de lazer ${ }^{31}$.

Um estudo retrospectivo de natureza documental, desenvolvido em instituição de ensino superior do município de João Pessoa, Paraíba ${ }^{26}$, e outro, de coorte prospectiva, realizado com 1.161 trabalhadores em Madri ${ }^{22}$, convergem quanto à informação sobre a relação entre nível de instrução escolar e ausência no trabalho. O estudo de coorte ${ }^{22}$ revelou que a duração média da incapacidade temporal por contingências comuns nos afastamentos por transtornos de ansiedade foi de 83 dias. Apontou, ainda, que os trabalhadores tinham idade superior a 35 anos, eram do sexo feminino, possuíam menor nível de instrução e alguma comorbidade.

Em relação à presença de comorbidades, há maior risco de afastamento do trabalho quando existem concomitantemente ansiedade e depressão. Convém destacar que o impacto é negativo tanto para o trabalhador como para seu empregador, em virtude do absenteísmo, da queda de produtividade e da alta rotatividade de profissionais. Ressalta-se, ainda, o elevado custo para o sistema de proteção social $^{22}$.

Neste sentido, duas pesquisas realizadas no Reino Unido, nos anos de 2011 e 2014, relataram que a perda associada de produtividade e o pagamento de benefícios por invalidez acarretaram custos consideráveis para as economias de muitos países desenvolvidos $^{20}$, e que a alta prevalência de transtornos ansiosos como motivo dos afastamentos laborais está associada a elevadas taxas de desemprego, diminuição do bem-estar e maior dependência da assistência pública $^{24}$. 
Assim, os resultados obtidos nesta revisão não apenas reforçam o que a OMS e a OIT já previam como tendência no campo da saúde pública e do trabalho - $\mathrm{o}$ aumento dos casos de transtornos mentais, incluindo os de ansiedade ${ }^{1}$-, como também alertam para a necessidade de reforçar as concepções de saúde mental e de trabalho como indissociáveis entre si na conjuntura produtiva atual, a fim de evitar o adoecimento mental e a ausência no trabalho. Vale ressaltar que a não utilização de outras bases, além das aqui pesquisadas, pode ter trazido limitações aos resultados deste estudo.

\section{Conclusão}

As evidências apontam para uma alta prevalência dos transtornos ansiosos como causa dos afastamentos do trabalho e da alta demanda de custos com auxílio-doença. O processo de afastamento do trabalhador gera impacto negativo em sua vida, sobretudo quando é decorrente das manifestações psicopatológicas dos transtornos ansiosos, que impõem limitações às suas atividades cotidianas, reduzem suas potencialidades no trabalho e interferem na convivência familiar e social.
Portanto, a partir dos estudos analisados pode-se afirmar a relação entre as condições de trabalho e a ocorrência dos transtornos de ansiedade. Assim, com o reconhecimento dessa relação será possível realizar as intervenções necessárias para prevenir ou reduzir tais agravos, diminuindo o número dos afastamentos laborais.

Por esse motivo, ressalta-se a necessidade de ampliar o olhar sobre o afastamento por transtornos de ansiedade para além da urgência de reduzir custos financeiros, compreendendo também o custo humano gerado pelo adoecimento por transtornos mentais.

Desta forma, conhecer o perfil de adoecimento do trabalhador é indispensável nesse processo, uma vez que possibilita o diagnóstico situacional, contribui para o reconhecimento dos fatores de risco e auxilia nas estratégias de intervenção direcionadas à melhoria das condições de trabalho, na qualidade de vida e na redução do afastamento laboral.

Ressalta-se a lacuna de estudos nacionais sobre o tema em questão, sendo importante realizar novos estudos, com níveis de evidência mais robustos, capazes de explorar com maior profundidade os afastamentos laborais devido aos transtornos de ansiedade.

\section{Contribuições de autoria}

Ribeiro HKP foi responsável por construir o projeto, realizar a pesquisa, consolidar e discutir os resultados; Santos JDM, pela realização da pesquisa e consolidação dos resultados; Silva MG, Medeiro FDA, Fernandes MA, pela discussão dos resultados e elaboração da versão final do artigo. Todos os autores contribuíram para a aprovação da versão final do manuscrito.

\section{Referências}

1. World Health Organization. International Labour Organization. Mental health and work: impact, issues and good practices. Geneva: WHO; 2000.

2. Landsbergis PA, Grzywacz JG, LaMontagne AD. Work organization, job insecurity, and occupational health disparities. Am J Ind Med. 2014;57(5):495-515.

3. Cordeiro TMSC, Mattos AIS, Cardoso MCB, Santos KOB, Araújo TM. Notificações de transtornos mentais relacionados ao trabalho entre trabalhadores na Bahia: estudo descritivo, 20072012. Epidemiol Serv Saúde. 2016;25(2):363-72.

4. Baasch D, Trevisan RL, Cruz RM. Epidemiological profile of public servants absent from work due to mental disorders from 2010 to 2013. Ciênc Saúde Coletiva. 2017;22(5):1641-50.

5. Carvalho DB, Araújo TM, Bernardes KO. Transtornos mentais comuns em trabalhadores da
Atenção Básica à Saúde. Rev Bras Saude Ocup. 2016;41:e17.

6. Silva-Junior JS, Fisher FM. Sickness absence due to mental disorders and psychosocial stressors at work. Rev Bras Epidemiol. 2015;18(4):735-44.

7. Leite AF, Nogueira JAD. Fatores condicionantes de saúde relacionados ao trabalho de professores universitários da área da saúde: uma revisão integrativa. Rev Bras Saude Ocup. 2017;42: e6.

8. Forattini CD, Lucena C. Adoecimento e sofrimento docente na perspectiva da precarização do trabalho. Laplage em Revista. 2015;1(2):32-47.

9. Araújo TM, Mattos AIS, Almeida MMG, Santos KOB. Aspectos psicossociais do trabalho e transtornos mentais comuns entre trabalhadores da saúde: contribuições da análise de modelos combinados. Rev Bras Epidemiol. 2016;19(3):645-657. 
10. Estorce TP, Kurcgan TP. Sick leave and nursing personnel management. Rev Esc Enferm USP. 2011;45(5):1199-205.

11. Guimarães AMV, Neto ACS, Vilar ATS, Almeida BGC, Albuquerque CMF, Fermoseli AFO. Transtornos de ansiedade: um estudo de prevalência sobre as fobias específicas e a importância da ajuda psicológica. Semina Cienc Biol Saude. 2015;3(1):115-28.

12. Duval ER, Javanbakht A, Liberzon I. Neural circuits in anxiety and stress disorders: a focused review. Ther Clin Risk Manag. 2015;11:115-26.

13. Santana LL, Sarquis LMM, Brey C, Miranda FMA, Felli VEA. Absenteeism due to mental disorders in health professionals at a hospital in southern Brazil. Rev Gaúcha Enferm. 2016;37(1):e53485.

14. Mendes KDS, Silveira RCCP, Galvão CM. Revisão integrativa: método de pesquisa para a incorporação de evidências na saúde e na enfermagem. Texto Contexto Enferm. 2008;17(4):758-64.

15. Nieuwenhuijsen K, Verbeek JH, De Boer AG, Blonk RW, Van Dijk FJ. Predicting the duration of sickness absence for patients with common mental disorders in occupational health care. Scand J Work Environ Health. 2006;32(1):67-74.

16. Rymaszewska J, Dziełak K, Kiejna A. Incapacity for work and disability pensions in persons with mental disorders. Psychiatr Pol. 2007;41(2):171-80.

17. Andrea H, Bültmann U, Van Amelsvoort LG, Kant Y. The incidence of anxiety and depression among employees-the role of psychosocial work characteristics. Depress Anxiety. 2009;26(11):1040-8.

18. Siano AK, Ribeiro LC, Ribeiro MS. Análise comparativa do registro médico-pericial do diagnóstico de transtornos mentais de segurados do Instituto Nacional do Seguro Social requerentes de auxílio-doença. J Bras Psiquiatr. 2010;59(2):131-8

19. Muschalla B, Linden M, Olbrich D. The relationship between job-anxiety and trait-anxiety: a differential diagnostic investigation with the Job-Anxiety-Scale and the State-Trait-AnxietyInventory. J Anxiety Disord. 2010;24(3):366-71.

20. Henderson M, Harvey SB, Overland S, Mykletun A, Hotopf M. Work and common psychiatric disorders. J R Soc Med. 2011;104(5):198-207.

21. Flach PA, Groothoff JW, Krol B, Bültmann U. Factors associated with first return to work and sick leave durations in workers with common mental disorders. Eur J Public Health. 2011;22(3):440-5.

22. Romero CC, Muñoz PM, Aguado LQ, Moraga MR, Labandera CF, Bonacho EC. Predictores de la duración de la incapacidad temporal por contingências comunes en los trastornos de ansiedad. Gac Sanit. 2013;27(1):40-6.

23. Wedegaertner F, Arnhold-Kerri S, Sittaro NA, Bleich S, Geyer S, Lee WE. Depressionand anxiety-related sick leave and the risk of permanent disability and mortality in the working population in Germany: a cohort study. BMC Public Health. 2013;13(145):1-10.

24. Combs H, Markman J. Anxiety Disorders in Primary Care. Med Clin North Am. 2014;98(5):1007-23.

25. Niedhammer I, Malard L, Chastang JF. Occupational factors and subsequent major depressive and generalized anxiety disorders in the prospective French national SIP study. BMC Public Health. 2015;15(200):1-11.

26. Batista JBV, Carlotto MS, Oliveira MN, Zaccara AAL, Barros EO, Duarte MCS. Mental disorders in university teachers: study in a service of medical investigation. J res: fundam care online. 2016;8(2):4538-48.

27. Den Boeft M, Twisk JWR, Hoekstra T, Terluin B, Penninx BW, van der Wouden JC, et al. Medically unexplained physical symptoms and work functioning over 2 years: their association and the influence of depressive and anxiety disorders and job characteristics. BMC Fam Pract. 2016;17(46):1-8.

28. DeSousa DA, Moreno AL, Gauer G, Manfro GG, Koller SH. Revisão sistemática de instrumentos para avaliação de ansiedade na população brasileira. Avaliação Psicológica. 2013;12(3):397-410.

29. Assunção AA, Lima EP, Guimarães MDC. Transtornos mentais e inserção no mercado de trabalho no Brasil: um estudo multicêntrico nacional. Cad Saúde Pública. 2017;33(3):e00166815.

30. Carreiro GSP, Ferreira Filha MO, Lazarte R, Silva AO, Dias MD. O processo de adoecimento mental do trabalhador da Estratégia Saúde da Família. Rev Eletr Enf. 2013;15(1):146-55.

31. Primo GMG, Pinheiro TMM, Sakurai E. Absenteísmo por doença em trabalhadores de uma organização hospitalar pública e universitária. Rev Med Minas Gerais. 2010; 20(2 Supl 2):S47-S58. 